\title{
The knowledge and awareness about among married women in urban field practice area of Rama medical college hospital and research centre, Mandhana, Kanpur
}

\author{
Pankaj Kumar ${ }^{1}$, Bandana Kumari²* \\ ${ }^{1}$ Assistant Professor. Department of Community Medicine, Lord Buddha Koshi Medical College \& Hospital, Bihar, INDIA. \\ ${ }^{2}$ Medical Officer, Sub Divisional Hospital, Hilsa, Dist- Nalanda, Bihar, INDIA. \\ Email: pankajdr76@gmail.com.
}

\begin{abstract}
Background: A multi-pronged strategy aimed at sustained IEC efforts focusing on safety and beneficial effects of various contraceptives, clearing of misconception about side effects of contraceptives by family planning counselors and equal involvement of both husband and wife as one unit should be able to bring out an outcome favourable in terms of contraception use. Methods: The study of factors influencing family planning practices was carried out among 350 married women aged 18-49 years in the urban field practice area of Rama Medical College Hospital and Research Centre, Kanpur during the period of January 2017 to December 2018. Results: In the study group, majority 135(38.6\%) were aged between 26-33 years of age. Majority 309(88.3\%) were Hindus, 14(4\%) were Muslims and 13(3.7\%) were Christians in the study. Majority 121(34.6\%) had secondary level of education, 41(11.7\%) were illiterates, 16(4.6\%) were Post Graduate. Majority $174(49.7 \%)$ of current users have accessed contraception from Urban health center, $80(22.9 \%)$ from private pharmacy and 96(27.4\%) from tertiary level hospital and $264(75.4 \%)$ had no fear and $86(24.6 \%)$ had fear from the side effect of contraceptive. 187(53.4\%) women know about emergency contraceptive and 163(46.6\%) do not know about emergency contraceptive . Conclusion: In order to improve contraceptive use what we need today is multiple resources to educate couples, their parents, family members and society too, so what we can reach upto masses. Women must be made aware about their right i.e, protecting their own health . Good counseling practices along with clinical work are the need of time, for these women should be educated, be economical independent.
\end{abstract}

Key Word: contraceptive methods.

*Address for Correspondence:

Dr Bandana Kumari, Medical Officer, Sub divisional Hospital, HILSA, Dist- Nalanda, Bihar, INDIA.

Email: pankajdr76@gmail.com

Received Date: 08/12/2019 Revised Date: 20/01/2020 Accepted Date: 03/02/2020

DOI: https://doi.org/10.26611/10111511

This work is licensed under a Creative Commons Attribution-NonCommercial 4.0 International License. $(\boldsymbol{C c})$ EY-NC

\begin{tabular}{|l|l|}
\hline \multicolumn{2}{|c|}{ Access this article online } \\
\hline Quick Response Code: & Website: \\
\hline & www.medpulse.in \\
& \\
\hline
\end{tabular}

\section{INTRODUCTION}

India is the second most populous country in the world, next only to China, where as seventh in land area. With only $2.4 \%$ of the world's land area, India is supporting about $16.87 \%$ of the world's population. The population of India 1.21 billion in 2011, (2001 is 102.8 crores), average exponential growth rate $1.95 \%$ in 2001 , and the decadal growth of population is $17.6 \%$ in $2011(21.52 \%$ in 2001). ${ }^{1}$ The death rate declines still further and the birth rate tends to fall. The population continues to grow because births exceed deaths. India has entered this late expanding phase. At the beginning of the century, Dr Pyare Krishnan wattal (1916) observed that our country's increasing birth rate was a social danger. In his words, "if we want to go to the root of it (increasing birth rate), we must look at the causes that give rise to this high birth rate, much more seriously than to the secondary causes that give rise to high death rate". 2 "Women and men in many countries still lack adequate access to contraceptives, unless they are given the option of controlling their fertility, severe environmental and health problems loon in the coining century throughout 
large parts of the world"3. More than 100 million women in developing countries or about $17 \%$ of all married women would prefer to avoid a pregnancy but are not using any form of family planning ${ }^{4}$. India was the first country in the world to formulate the national family planning programme in the year 1952 with the objective of "reducing the birth rate to the extent necessary to stabilize the population at a level consistent with requirement of national economy" 5 . The family planning programme has undergone transformation in terms of policy and actual programme implementation. There occurred a gradual shift from clinical approach to the reproductive child health approach and further the National Population Policy (NPP) 2000 brought a holistic and a target free approach which helped in reduction of fertility. The objectives, strategies and activities of the Family Planning division are designed and operated towards achieving the family welfare goals and objectives stated in various policy documents (NPP: National Population Policy 2000, NHP: National Health Policy 2002 and NRHM: National Rural Health Mission) and to honour the commitments of the Government of India (including ICPD: International Conference on Population and Development, MDG: Millennium Development Goals, FP 2020 Summit and others). ${ }^{6}$ The family welfare programme is mainly based on a "Cafeteria approach"; where by a number of methods of contraception are offered to the eligible couples. The national family welfare programme, in India, has traditionally sought "to promote responsible and planned parenthood through voluntary and free choice of family planning methods, best suited to individual acceptors". However, these methods have not been widely accepted by a large number of the eligible couples, as these methods do not meet their psycho-social requirements. Thus, inspite of various efforts on the government's part, the percentage of eligible couples using contraception (couple protection) rate is only $56.3 \%{ }^{7}$

\section{METHODS}

The study will be carried out among married women in the reproductive age group of 18-49 years in the field area of UHTC (urban health training centre, Kalyanpur) of Rama medical college hospital and research centre, Mandhana, Kanpur.

UHTC provides health care to 12 mohallas of ward 18 and 42 of urban area of kalyanpur, out of which 03 mohallas namely Kalyanpur Khurd, Janakipuram and R.K Puram are selected for study by systematic random sampling method.

It is a community based cross-sectional study. Urban field practice area (U.H.T.C, Kalyanpur) of Rama Medical College hospital and research center, Mandhana, Kanpur.
Married women in the reproductive age group of 18-49 years, residing in urban field practice area (U.H.T.C, Kalyanpur) of Rama Medical College hospital and research centre, Mandhana.

Inclusion Criteria :

- Women who are married, age of $18-49$ yrs.

- presumed to be sexually active.

- Pregnant and Postpartum amenorrhoeic women are also included

Exclusion Criteria :

- Those who are not willing to participate.

- Widow women

Study Period was January 2017 to December2018. And it was Systematic random sampling method.

The sample size is calculated by taking the prevalence rate of contraceptive use $(56.3 \%)$ at $5 \%$ significance level and $10 \%$ error 350 About $10 \%$ more than sample size interviewed) UHTC provides health care to 12 mohallas of ward 18 and 42 of urban area of kalyanpur, out of which 03 mohallas namely kalyanpur khurd, janakipuram and R.K puram having population of $3000+1500+1000$ respectively $=5500$ (As population of females aged 1849 is 220 per thousand )

Thus total female population 18-49 of above three mohallas $=5500 \times 220 / 1000=1210$

$1210 / 310=3.90$ rounded off to $\sim 4$ above age group females interviewed every $4^{\text {th }}$ house of all 03 study mohallas.

Data was processed on the software SPSS (Version 21.0), and Microsoft Excel 2010. Rates, ratios, proportions were calculated and cross tables with variables to ascertain 'association' were made. Statistical test for significance was applied on Chi-square distribution of the data analysis and of variance (ANOVA).

\section{RESULTS}

Observations of study are presented under following headings.

Part-A

1- Demographic profile of respondents and their statistical distribution $(\mathrm{N}=350)$.

2- Prevalence of contraceptive among various groups

3- Type of Contraceptive methods adopted among various groups.

4- Various factors affecting adoption of contraception.
a) Decision of husband
b) source of information about various contraceptive methods
c) availability of family welfare programme
d) fear of side effect

5- Knowledge of emergency contraceptive and no. of subjects using emergency contraceptive. 


\section{Part-B}

6- Awareness about injectable contraception.

7- Source of information about injectable contraceptive.

8- Side effects of injectable contraceptive among various age groups
9- Acceptability of injectable contraceptive among study subjects

10- Reasons for not using injectable contraceptive

11- Statistical analysis of variance (ANOVA).

\begin{tabular}{|c|c|c|c|c|c|c|c|c|c|}
\hline \multicolumn{2}{|c|}{$\mathbf{N}$} & \multirow[b]{2}{*}{ Mean } & \multirow{2}{*}{$\begin{array}{l}\text { Std. Error } \\
\text { of Mean }\end{array}$} & \multirow[b]{2}{*}{ Median } & \multirow[b]{2}{*}{ Mode } & \multirow{2}{*}{$\begin{array}{c}\text { Std. } \\
\text { Deviation }\end{array}$} & \multirow[b]{2}{*}{ Range } & \multirow[b]{2}{*}{ Minimum } & \multirow[b]{2}{*}{ Maximum } \\
\hline Valid & Missing & & & & & & & & \\
\hline 350 & 0 & 32.559 & .42123 & 30.0000 & 29.00 & 7.88042 & 25.00 & 21.00 & 46.00 \\
\hline
\end{tabular}

Above table shows statistical analysis of age of respondents participated in present study. The mean age of respondents $(\mathrm{N}=350)$ is 32.559 years with minimum 21years and maximum 46 years, median 30 years (with standard error of mean 0.42123 and standard deviation 7.88042)

Table 2: Distribution of the study subject according to the Age

\begin{tabular}{ccc}
\hline Age group & Frequency & Percent \\
\hline $18-25$ & 67 & 19.1 \\
$26-33$ & 135 & 38.6 \\
$34-41$ & 83 & 23.7 \\
$42-49$ & 65 & 18.6 \\
\hline Total & 350 & 100.0 \\
\hline
\end{tabular}

In the above table it is observed that majority of the women were between 26 and 33 years age group which is the most crucial in the reproductive span. Among the 350 women, 135 (38.6\%) were between 26 and 33 years, $83(23.7 \%)$ were between 34 and 41 years, 67 (19.1\%) were between 18 and 25 years, 65 (18.6\%) were between 42 and 49 years of age.

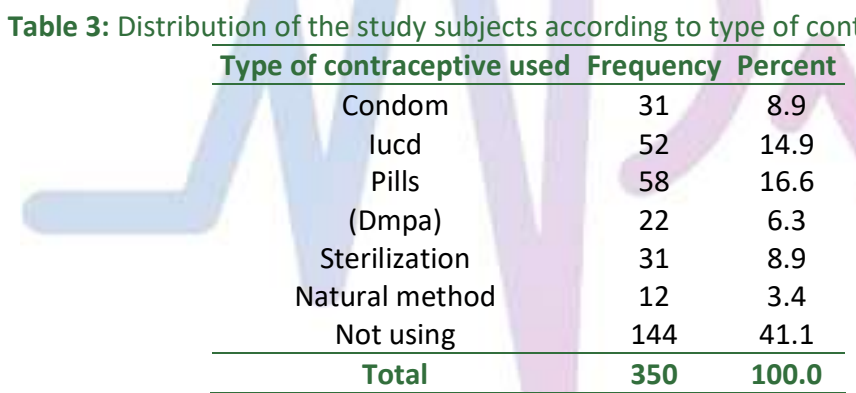

The above table show 58(16.6\%) female was using OCP, 52(14.9\%) was using IUCD, 31(8.9\%) was using condom 22 $(6.3 \%)$ was using DMPA, $31(8.9 \%)$ has got sterilization and $12(3.4 \%)$ was practiced natural method and $144(41.1 \%)$ was not using any type of contraception.

Table 4: Distribution of the study subjects according to the Type of contraception using currently in different age group

\begin{tabular}{|c|c|c|c|c|c|c|c|c|}
\hline \multicolumn{9}{|c|}{ Type of contraceptive used } \\
\hline Age group & Condom & IUCD & Pills & (DMPA) & Sterilization & $\begin{array}{l}\text { Natural } \\
\text { method }\end{array}$ & Not using & Total \\
\hline & 3 & 10 & 14 & 2 & 7 & 0 & 31 & 67 \\
\hline \multirow[t]{2}{*}{$18-25$} & $4.50 \%$ & $14.90 \%$ & $20.90 \%$ & $3.00 \%$ & $10.40 \%$ & $0.00 \%$ & $46.30 \%$ & $100 \%$ \\
\hline & 6 & 21 & 30 & 11 & 12 & 4 & 51 & 135 \\
\hline \multirow[t]{2}{*}{$26-33$} & $4.40 \%$ & $15.60 \%$ & $22.20 \%$ & $8.10 \%$ & $8.90 \%$ & $3.00 \%$ & $37.80 \%$ & $100 \%$ \\
\hline & 12 & 12 & 9 & 5 & 5 & 3 & 37 & 83 \\
\hline \multirow[t]{2}{*}{$34-41$} & $14.50 \%$ & $14.50 \%$ & $10.80 \%$ & $6.00 \%$ & $6.00 \%$ & $3.60 \%$ & $44.60 \%$ & $100 \%$ \\
\hline & 10 & 9 & 5 & 4 & 7 & 5 & 25 & 65 \\
\hline \multirow[t]{2}{*}{$42-49$} & $15.40 \%$ & $13.80 \%$ & $7.70 \%$ & $6.20 \%$ & $10.80 \%$ & $7.70 \%$ & $38.50 \%$ & $100 \%$ \\
\hline & 31 & 52 & 58 & 22 & 31 & 12 & 144 & 350 \\
\hline Total & $8.90 \%$ & $14.90 \%$ & $16.60 \%$ & $6.30 \%$ & $8.90 \%$ & $3.40 \%$ & $41.10 \%$ & $100 \%$ \\
\hline
\end{tabular}

In the above table in age group 18-25 maximum 14 (20.9\%) were using OCP, 10 (14.9\%) were using IUCD and no one were practiced natural method. In age group 26-33, maximum 30(22.2\%) were using OCP and minimum 4(3\%) were using natural method of contraception. 
Table 5: Distribution of study subjects according to the educational status and current type of contraception

\begin{tabular}{|c|c|c|c|c|c|c|c|c|}
\hline \multirow[b]{2}{*}{$\begin{array}{c}\text { Educational } \\
\text { status }\end{array}$} & \multicolumn{7}{|c|}{ Type of contraceptive used } & \multirow[b]{2}{*}{ Total } \\
\hline & Condom & IUCD & Pills & $\begin{array}{l}\text { Injectable } \\
\text { (DMPA) }\end{array}$ & Sterilization & $\begin{array}{l}\text { Natural } \\
\text { method }\end{array}$ & Not using & \\
\hline \multirow{3}{*}{ Illiterate } & 4 & 7 & 6 & 2 & 6 & 2 & 14 & 41 \\
\hline & $9.80 \%$ & $17.10 \%$ & $14.60 \%$ & $4.90 \%$ & $14.60 \%$ & $4.90 \%$ & $34.10 \%$ & $100 \%$ \\
\hline & 4 & 7 & 9 & 1 & 3 & 4 & 17 & 45 \\
\hline \multirow[t]{2}{*}{ Primary } & $8.90 \%$ & $15.60 \%$ & $20.00 \%$ & $2.20 \%$ & $6.70 \%$ & $8.90 \%$ & $37.80 \%$ & $100 \%$ \\
\hline & 8 & 11 & 8 & 8 & 7 & 2 & 25 & 69 \\
\hline \multirow[t]{2}{*}{ Middle } & $11.60 \%$ & $15.90 \%$ & $11.60 \%$ & $11.60 \%$ & $10.10 \%$ & $2.90 \%$ & $36.20 \%$ & $100 \%$ \\
\hline & 10 & 17 & 22 & 4 & 8 & 3 & 57 & 121 \\
\hline \multirow[t]{2}{*}{ Secondary } & $8.30 \%$ & $14.00 \%$ & $18.20 \%$ & $3.30 \%$ & $6.60 \%$ & $2.50 \%$ & $47.10 \%$ & $100 \%$ \\
\hline & 4 & 7 & 8 & 3 & 6 & 0 & 18 & 46 \\
\hline \multirow[t]{2}{*}{ Higher secondary } & $8.70 \%$ & $15.20 \%$ & $17.40 \%$ & $6.50 \%$ & $13.00 \%$ & $0.00 \%$ & $39.10 \%$ & $100 \%$ \\
\hline & 0 & 1 & 3 & 2 & 0 & 0 & 6 & 12 \\
\hline Graduate & $0.00 \%$ & $8.30 \%$ & $25.00 \%$ & $16.70 \%$ & $0.00 \%$ & $0.00 \%$ & $50.00 \%$ & $100 \%$ \\
\hline \multirow{2}{*}{ Post graduate } & 1 & 2 & 2 & 2 & 1 & 1 & 7 & 16 \\
\hline & $6.30 \%$ & $12.50 \%$ & $12.50 \%$ & $12.50 \%$ & $6.30 \%$ & $6.30 \%$ & $43.80 \%$ & $100 \%$ \\
\hline & 31 & 52 & 58 & 22 & 31 & 12 & 144 & 350 \\
\hline Total & $8.90 \%$ & $14.90 \%$ & $16.60 \%$ & $6.30 \%$ & $8.90 \%$ & $3.40 \%$ & $41.10 \%$ & $100 \%$ \\
\hline
\end{tabular}

In the above table, in illiterate group maximum $7(17.1 \%)$ were using IUCD, in primary group maximum $9(20 \%)$ were using OCP, in middle maximum $11(15.9 \%)$ were using IUCD, in secondary group maximum $22(18.2 \%)$ were using OCP, in higher secondary group maximum $8(17.4 \%)$ were using OCP,. In graduate group maximum $3(25 \%)$ were using OCP as contraceptive method.

Table 6: Distribution of study subjects according to the occupational status and current type of contraception

\begin{tabular}{|c|c|c|c|c|c|c|c|c|}
\hline \multirow[b]{2}{*}{$\begin{array}{l}\text { occupational } \\
\text { status }\end{array}$} & \multicolumn{7}{|c|}{ Type of contraceptive used } & \multirow[b]{2}{*}{ Total } \\
\hline & Condom & IUCD & Pills & $\begin{array}{l}\text { Injectable } \\
\text { (DMPA) }\end{array}$ & $\begin{array}{l}\text { Steriliz- } \\
\text { ation }\end{array}$ & $\begin{array}{l}\text { Natural } \\
\text { method }\end{array}$ & Not using & \\
\hline & 23 & 37 & 39 & 16 & 20 & 11 & 95 & 241 \\
\hline \multirow[t]{2}{*}{ Home maker } & $9.50 \%$ & $15.40 \%$ & $16.20 \%$ & $6.60 \%$ & $8.30 \%$ & $4.60 \%$ & $39.40 \%$ & $100 \%$ \\
\hline & 3 & 7 & 8 & 3 & 5 & 0 & 21 & 47 \\
\hline \multirow[t]{2}{*}{ Unskilled } & $6.40 \%$ & $14.90 \%$ & $17.00 \%$ & $6.40 \%$ & $10.60 \%$ & $0.00 \%$ & $44.70 \%$ & $100 \%$ \\
\hline & 4 & 6 & 5 & 1 & 3 & 1 & 14 & 34 \\
\hline \multirow[t]{2}{*}{ Semiskilld } & $11.80 \%$ & $17.60 \%$ & $14.70 \%$ & $2.90 \%$ & $8.80 \%$ & $2.90 \%$ & $41.20 \%$ & $100 \%$ \\
\hline & 1 & 1 & 2 & 2 & 2 & 0 & 6 & 14 \\
\hline \multirow[t]{2}{*}{ Skilled } & $7.10 \%$ & $7.10 \%$ & $14.30 \%$ & $14.30 \%$ & $14.30 \%$ & $0.00 \%$ & $42.90 \%$ & $100 \%$ \\
\hline & 0 & 1 & 4 & 0 & 1 & 0 & 8 & 14 \\
\hline \multirow[t]{2}{*}{ Professioal } & $0.00 \%$ & $7.10 \%$ & $28.60 \%$ & $0.00 \%$ & $7.10 \%$ & $0.00 \%$ & $57.10 \%$ & $100 \%$ \\
\hline & 31 & 52 & 58 & 22 & 31 & 12 & 144 & 350 \\
\hline Total & $8.90 \%$ & $14.90 \%$ & $16.60 \%$ & $6.30 \%$ & $8.90 \%$ & $3.40 \%$ & $41.10 \%$ & $100 \%$ \\
\hline
\end{tabular}

In the above table among the home maker maximum 39(16.2\%) were using OCP, among unskilled 17\% were using OCP, overall OCP were used maximum as contraceptive method.

Table 7: Distribution of study subjects according to the Socioeconomic status and current type of contraception

\begin{tabular}{|c|c|c|c|c|c|c|c|c|}
\hline \multirow[b]{2}{*}{$\begin{array}{c}\text { socioeconomic } \\
\text { status }\end{array}$} & \multicolumn{7}{|c|}{ Type of contraceptive used } & \multirow[b]{2}{*}{ Total } \\
\hline & condom & IUCD & pills & $\begin{array}{l}\text { injectable } \\
\text { (DMPA) }\end{array}$ & $\begin{array}{l}\text { sterili- } \\
\text { zation }\end{array}$ & $\begin{array}{l}\text { natural } \\
\text { method }\end{array}$ & not using & \\
\hline & 3 & 0 & 11 & 4 & 1 & 4 & 19 & 42 \\
\hline Upper class & $7.10 \%$ & $0.00 \%$ & $26.20 \%$ & $9.50 \%$ & $2.40 \%$ & $9.50 \%$ & $45.20 \%$ & $100 \%$ \\
\hline \multirow{2}{*}{ Upper middle } & 4 & 11 & 4 & 4 & 1 & 0 & 11 & 35 \\
\hline & $11.40 \%$ & $31.40 \%$ & $11.40 \%$ & $11.40 \%$ & $2.90 \%$ & $0.00 \%$ & $31.40 \%$ & $100.00 \%$ \\
\hline \multirow[t]{3}{*}{ Lower middle } & 9 & 16 & 18 & 6 & 10 & 5 & 41 & 105 \\
\hline & $8.60 \%$ & $15.20 \%$ & $17.10 \%$ & $5.70 \%$ & $9.50 \%$ & $4.80 \%$ & $39.00 \%$ & $100 \%$ \\
\hline & 8 & 11 & 15 & 4 & 14 & 1 & 41 & 94 \\
\hline \multirow[t]{2}{*}{ Upper lower } & $8.50 \%$ & $11.70 \%$ & $16.00 \%$ & $4.30 \%$ & $14.90 \%$ & $1.10 \%$ & $43.60 \%$ & $100 \%$ \\
\hline & 7 & 14 & 10 & 4 & 5 & 2 & 32 & 74 \\
\hline \multirow[t]{2}{*}{ Lower } & $9.50 \%$ & $18.90 \%$ & $13.50 \%$ & $5.40 \%$ & $6.80 \%$ & $2.70 \%$ & $43.20 \%$ & $100 \%$ \\
\hline & 31 & 52 & 58 & 22 & 31 & 12 & 144 & 350 \\
\hline Total & $8.90 \%$ & $14.90 \%$ & $16.60 \%$ & $6.30 \%$ & $8.90 \%$ & $3.40 \%$ & $41.10 \%$ & $100 \%$ \\
\hline
\end{tabular}


Above table show in upper class maximum 11(26.2\%) were using oral contraceptive pills. sterilization methods were maximum $14.9 \% \%$ in upper lower class. IUCD were maximum $31.4 \%$ used in upper middle class. Condom uses maximum in upper middle class. Injectable maximum used by upper class.

Table 8: Distribution of study subjects according to Religion and current use of contraception

\begin{tabular}{cccc}
\hline & \multicolumn{2}{c}{ Are you using contraceptive method currently } & \multicolumn{2}{c}{ Total } \\
\cline { 2 - 4 } Religion & Yes & No & \\
\cline { 2 - 4 } Hindu & 180 & 129 & 309 \\
& $58.3 \%$ & $41.7 \%$ & $100.0 \%$ \\
Muslim & 5 & 9 & 14 \\
& $35.7 \%$ & $64.3 \%$ & $100.0 \%$ \\
Christian & 10 & 3 & 13 \\
& $76.9 \%$ & $23.1 \%$ & $100.0 \%$ \\
Others & 11 & 3 & 14 \\
Total & $78.6 \%$ & $21.4 \%$ & $100.0 \%$ \\
\hline & 206 & 144 & 350 \\
& $58.9 \%$ & $41.1 \%$ & $100.0 \%$ \\
\hline
\end{tabular}

In the above table maximum 180 (58.3\%) hindu family were using contraceptive in study population. In muslim $35.7 \%$ were using and $64.3 \%$ were not using any contraception. Among chtistian $76.9 \%$ were using and $21.4 \%$ were not using any contraceptive method.

Table 9: Distribution of the study subjects according to the husband's opinion towards contraception

\begin{tabular}{ccc}
\hline $\begin{array}{c}\text { Husband opinion towards } \\
\text { contraception }\end{array}$ & Frequency & Percent \\
\hline Agree & 250 & 71.4 \\
Disagree & 100 & 28.6 \\
\hline Total & 350 & 100.0 \\
\hline
\end{tabular}

In the above table, it was observed that majority 250 (71.4\%) husbands agree for use of contraception and $100(28.6 \%)$ disagrees for contraception.

Table 10: Distribution of study subjects according to fear of side effect from contraceptive

\begin{tabular}{cccc}
\hline Fear of side effects & Frequency & Percent \\
\hline Yes & 86 & 24.6 \\
No & 264 & 75.4 \\
\hline Total & 350 & 100.0 \\
\hline
\end{tabular}

In the above table 264(75.4\%) had no fear and 86(24.6\%) had fear from the side effect of contraceptive.

Table 11: Distribution of study subjects according to knowledge about injectable contraceptive

\begin{tabular}{ccc}
\hline Awareness about Injectable contraceptives & Frequency & Percent \\
\hline Yes & 138 & 39.4 \\
No & 212 & 60.6 \\
\hline Total & 350 & 100.0 \\
\hline
\end{tabular}

Above table shows only138(39.4\%) women know about injectable contraceptive and 212(60.6\%) do not know about injectable contraceptive.

Table 12: Distribution of study subjects according to acceptability of DMPA

\begin{tabular}{ccccc}
\hline Acceptability of DMPA & Frequency & Percent & Valid Percent & Cumulative Percent \\
\hline Own decision & 3 & .9 & .9 & .9 \\
After councelling & 19 & 5.4 & 5.4 & 6.3 \\
Unacceptable & 328 & 93.7 & 93.7 & 100.0 \\
\hline Total & 350 & 100.0 & 100.0 & \\
\hline
\end{tabular}

Above table show acceptability of DMPA is $6.3 \%$, of which $0.9 \%$ users had own decision, and $5.4 \%$ accepted after counseling.

Table 13: Distribution of study subjects according to reason for not using injectable contraceptive

\begin{tabular}{ccc}
\hline Reason for not using IC & Frequency & Percent \\
\hline Fear of side effect & 122 & 34.9 \\
Unacceptable to family members & 16 & 4.6 \\
Lack of awareness about IC & 212 & 60.6 \\
\hline Total & 350 & 100.0 \\
\hline
\end{tabular}


Above table shows the main cause of not using injectable contraceptive was lack of awareness in $212(60.6 \%)$ women. $34.9 \%$ women had fear of side effect.

\begin{tabular}{|c|c|c|c|c|c|c|}
\hline & & Sum of Squares & df & Mean Square & $\mathbf{F}$ & Sig. \\
\hline \multirow{3}{*}{ Age of respondent } & Between Groups & 170.219 & 1 & 170.219 & 2.755 & .098 \\
\hline & Within Groups & 21503.054 & 348 & 61.790 & & \\
\hline & Total & 21673.272 & 349 & & & \\
\hline \multirow{3}{*}{ Religion } & Between Groups & 1.119 & 1 & 1.119 & 2.268 & .133 \\
\hline & Within Groups & 171.670 & 348 & .493 & & \\
\hline & Total & 172.789 & 349 & & & \\
\hline \multirow{3}{*}{ Educational status } & Between Groups & 7.657 & 1 & 7.657 & 3.454 & .064 \\
\hline & Within Groups & 771.498 & 348 & 2.217 & & \\
\hline & Total & 779.154 & 349 & & & \\
\hline \multirow{3}{*}{ Occupational status } & Between Groups & 4.894 & 1 & 4.894 & 4274 & 039 \\
\hline & Within Groups & 398.481 & 348 & 1.145 & $4.2 / 4$ & .039 \\
\hline & Total & 403.374 & 349 & & & \\
\hline \multirow{3}{*}{ Members in family } & Between Groups & 13.617 & 1 & 13.617 & & \\
\hline & Within Groups & 429.241 & 348 & 1.233 & 11.039 & .001 \\
\hline & Total & 442.857 & 349 & & & \\
\hline \multirow{2}{*}{$\begin{array}{l}\text { Interval between } \\
\text { marriage and first }\end{array}$} & Between Groups & 8.999 & 1 & 8.999 & 19.169 & .000 \\
\hline & Within Groups & 163.370 & 348 & .469 & & \\
\hline pregnancy & Total & 172.369 & 349 & & & \\
\hline \multirow{4}{*}{ Desired family size } & Between Groups & .858 & 1 & .858 & 3458 & 064 \\
\hline & Within Groups & 86.297 & 348 & .248 & 3.458 & .064 \\
\hline & Total & 87.154 & 349 & & & \\
\hline & Between Groups & 1.403 & 1 & 1.403 & & \\
\hline \multirow{2}{*}{$\begin{array}{c}\text { Did you have any } \\
\text { abortion }\end{array}$} & Within Groups & 75.466 & 348 & .217 & 6.468 & .011 \\
\hline & Total & 76.869 & 349 & & & \\
\hline \multirow{3}{*}{$\begin{array}{c}\text { Have you any } \\
\text { fear of side effect } \\
\text { from }\end{array}$} & Between Groups & 4.089 & 1 & 4.089 & 23.415 & .000 \\
\hline & Within Groups & 60.779 & 348 & .175 & & \\
\hline & Total & 64.869 & 349 & & & \\
\hline Source of & Between Groups & 34.376 & 1 & 34.376 & 28.088 & .000 \\
\hline \multirow{2}{*}{$\begin{array}{c}\text { information about } \\
\text { injectable } \\
\text { contraceptive }\end{array}$} & Within Groups & 425.912 & 348 & 1.224 & & \\
\hline & Total & 460.289 & 349 & & & \\
\hline \multirow{3}{*}{$\begin{array}{c}\text { Do you know } \\
\text { about benefits of } \\
\text { contraception }\end{array}$} & Between Groups & .457 & 1 & .457 & 4.873 & .028 \\
\hline & Within Groups & 32.632 & 348 & .094 & & \\
\hline & Total & 33.089 & 349 & & & \\
\hline \multirow{3}{*}{$\begin{array}{c}\text { Husband opinion } \\
\text { towards } \\
\text { contraception }\end{array}$} & Between Groups & 1.214 & 1 & 1.214 & 6.016 & .015 \\
\hline & Within Groups & 70.215 & 348 & .202 & & \\
\hline & Total & 71.429 & 349 & & & \\
\hline
\end{tabular}

\section{DISCUSSION}

It is observed that majority of the women were between 26 and 33 years age group which is the most crucial in the reproductive span. Among the 350 women, 135 (38.6\%) were between 26 and 33 years, $83(23.7 \%)$ were between 34 and 41 years, $67(19.1 \%)$ were between 18 and 25 years, $65(18.6 \%)$ were between 42 and 49 years of age In India, study conducted in Uttar Pradesh, percentage distribution of married women constitute $21 \%$ were between 25-29 years, $20 \%$ were between 30-34 years, $19 \%$ were between $20-24$ years, $16 \%$ were between 35- 39 years, $11 \%$ were between $40-44$ years, $5 \%$ were between 15-19years ${ }^{8} .58(16.6 \%)$ female was using OCP, $52(14.9 \%)$ was using IUCD, 31(8.9\%) was using condom $22(6.3 \%)$ was using DMPA, 31(8.9\%) has got sterilization and $12(3.4 \%)$ was practicised natural method and $144(41.1 \%)$ was not using any type of contraception. In a rural population of Dehradun district, $49.86 \%$ women were using contraception of which $28.88 \%$ women were using permanent method of contraception 
while $18.17 \%$ were using contraceptives as a spacing method. In a study done in East Delhi, of the 59.8\% eligible couples who were using a contraceptive method, condom was the most common (33.4\%) method of contraception followed by other spacing methods $(32.3 \%)$ and tubectomy $(27.3 \%)^{6}$. In age group 18-25 maximum 14(20.9\%) were using OCP, 10(14.9\%) were using IUCD and no one were practiced natural method. In age group $26-33$,maximum $30(22.2 \%)$ were using OCP and minimum 4(3\%) were using natural method of contraception. Studies reveal that among those who underwent sterilization Tubectomy was more common $(74.6 \%)$ than vasectomy $(1.3 \%){ }^{54}$. The reasons for an early sterilization can be attributed to early age at marriage and early completion of family size of two to three children by the age of 22 years. These findings are on par with the study of A.M. Khan, which reports that delay in birth of first child is culturally unacceptable. Many of these women prefer to have sterilization by the age of 21 years ${ }^{9}$. In illiterate group maximum 7(17.1\%) were using IUCD, in primary group maximum $9(20 \%)$ were using OCP, in middle maximum $11(15.9 \%)$ were using IUCD, in secondary group maximum $22(18.2 \%)$ were using OCP, in higher secondary group maximum $8(17.4 \%)$ were using OCP. In graduate group maximum $3(25 \%)$ were using OCP as contraceptive method. In a similar study it was observed that $66.7 \%$ women using contraceptive had matric and above level of education. $16 \%$ had under metric level of education, $28 \%$ were illiterates ${ }^{[10]}$.

Among the home maker maximum 39(16.2\%) were using OCP, among unskilled $17 \%$ were using OCP, overall OCP were used maximum as contraceptive method. In upper class maximum 11(26.2\%) were using oral contraceptive pills. sterilization methods were maximum $14.9 \% \%$ in upper lower class. IUCD were maximum $31.4 \%$ used in upper middle class. Condom uses maximum in upper middle class. Injectable maximum used by upper class. Maximum 180 (58.3\%) Hindu family were using contraceptive in study population. In Muslim 35.7\% were using and $64.3 \%$ were not using any contraception. Among Christian $76.9 \%$ were using and $21.4 \%$ were not using any contraceptive method. Current use of family planning methods was similar for Hindus(43.1\%) and Muslims(44.1\%). However, ever use of contraception was found to be more among Muslims(72\%) than Hindus(50.6\%). Spacing methods were more popular among Muslim couples(84.3\%) compared to Hindus(71.7\%) whereas larger number of Hindu couples preferred terminal method(28.3\%) compared to Muslims (15.7\%). ${ }^{11}$ It was observed that majority 250(71.4\%) husbands agree for use of contraception and $100(28.6 \%)$ disagrees for contraception. In a similar study, $44.6 \%$ husbands agree for contraception ${ }^{12} .264(75.4 \%)$ had no fear and 86 (24.6\%) had fear from the side effect of contraceptive. A study shows $48.63 \%$ were contraceptive acceptors, $64.66 \%$ women were accepted permanent method of contraception. Among the temporary methods most commonly accepted was IUD by $19.28 \%$ women, commonest reason for not accepting contraceptives was desire of children in $25.85 \%$ women followed by fear of side effects in $16.34 \%$ women. ${ }^{13}$ Table shows only138(39.4\%) women know about injectable contraceptive and 212(60.6\%) do not know about injectable contraceptive. Data from NFHS-3 show that less than half $(49 \%)$ of all women have heard about injectable contraception. Acceptability of DMPA is $6.3 \%$, of which $0.9 \%$ users had own decision, and $5.4 \%$ accepted after counseling. The main cause of not using injectable contraceptive was lack of awareness in $212(60.6 \%)$ women. $34.9 \%$ women had fear of side effect.

\section{CONCLUSION}

In order to improve contraceptive use what we need today is multiple resourses to educate couples, their parents, family members and society too, so what we can reach upto masses. Women must be made aware about their right i.e, protecting their own health. Good counseling practices along with clinical work are the need of time, for these women should be educated, be economical independent. If we work as a team and provide door step counseling and services irrespective of caste, religion and socio-status, we can definitely achieve our goal of population stabilization.

\section{REFERENCES}

1. Park, k., Demography and family planning. Text book of preventive and social medicine, 22nd Edition: Jabalpur. $\mathrm{M} / \mathrm{s}$ Banarsidas Bhanot Publishers; 2013, Page no $443,445,447,454$.

2. Sinha, N.K., Family welfare. The state of India's Health, Voluntary Health Association of India, 1992:19 1.

3. Malcolm Potts; theunmet need for Family Planning. Scientific American, January 2000. 88-93.

4. Lori Ashford. Unmet Need for Family Planning: Recent trends and Their Implications for Programs. Population Reference Bureau unmetneed Fam PIan-Eng.pdf.

5. Kansal A, Chandra R, Kandpal S D. Negi K.S. Epidemiological Correlates of Contraceptive Prevalence in Rural Population of Dehradun District. Indian Journal of Community Medicine, April-June 2005, Vol 30, No2, 60-62.

6. Annual Report 2014-15, Chapter 9, Moh. F.W. Govt. Of India. 
7. NFHS 3 (National Family Health Survey), , Mumbai International Institute of Population Sciences, 2000. www.nfhsindia.com

8. Ahmed, Sohail Chand. Log Linear Models for Religious and Social Factors affecting the practice of Family Planning Methods in Lahore, Pakistan. Pak..J. stat. Oper. Res. Vol. ii No. 12006 pp (35-44).

9. Saha S P. Trends in socio demographic characteristics of tubectomy acceptors in rural areas of West Bengal. Indian journal of Public Health 1981 3:102.

10. USAID family planning need during the first two years postpartum in Bihar, India.
11. Family planning differentials among religious group . by Nazish Rasheed. Dept. of community medicine Hamdard institute of medical sciences and research, new delhi.

12. Rebha sabharwal. Factors associated with contraception in India. Center for Population Dynamics. Arizona State University. Population Association of America 2009Extended abstract.

13. Indian journal of science and Technology vol. 4 no. 8 (Aug 2011). Study of contraceptive practices and reasons for not accepting contraceptive.

Source of Support: None Declared
Conflict of Interest: None Declared

Policy for Articles with Open Access:

Authors who publish with MedPulse International Journal of Community Medicine, Print ISSN: 2579-0862, Online ISSN: 2636-4743 agree to the following terms: Authors retain copyright and grant the journal right of first publication with the work simultaneously licensed under a Creative Commons Attribution License that allows others to share the work with an acknowledgement of the work's authorship and initial publication in this journal.

Authors are permitted and encouraged to post links to their work online (e.g., in institutional repositories or on their website) prior to and during the submission process, as it can lead to productive exchanges, as well as earlier and greater citation of published work. 